\section{Comparison of Pre- and Post-translational Expressions of COXIV-1 and MT-ATPase 6 Genes in Colorectal Adenoma-Carcinoma Tissues}

LaShanale Wallace $^{1}$, Anju M Cherian ${ }^{1}$, Paula Adamson ${ }^{1}$, Shahla Bari ${ }^{1}$, Saswati Banerjee ${ }^{1}$, Michael Flood ${ }^{1}$, Melvin Simien ${ }^{1}$, Xuebiao Yao ${ }^{2}$ and Felix 0 Aikhionbare $^{1^{*}}$

${ }^{1}$ Department of Medicine, Morehouse School of Medicine, 720 Westview Dr. SW Atlanta, GA 30310-1495, USA

${ }^{2}$ Department of Physiology, Morehouse School of Medicine, 720 Westview Dr. SW Atlanta, GA 30310-1495, USA

${ }^{*}$ Corresponding Author: Dr. Felix O Aikhionbare, Department of Medicine, Morehouse School of Medicine, 720 Westview Dr. SW Atlanta, GA 30310-1495, USA, Tel: (404)-752-1065; Fax: (404)-756-1328; E-mail: faikhionbare@msm.edu

Received date: August 03, 2018; Accepted date: September 14, 2018; Published date: September 18, 2018

Copyright: $@ 2018$ Wallace L, et al. This is an open-access article distributed under the terms of the Creative Commons Attribution License, which permits unrestricted use, distribution, and reproduction in any medium, provided the original author and source are credited.

\begin{abstract}
Objective: Colorectal cancer (CRC) develops from precancerous adenomatous polyps to malignant lesions of adenocarcinoma. Elucidating inhibition mechanisms for this route in patients with a risk of developing CRC is highly important for a potential diagnostic or prognostic marker. Differential expression of nuclear-encoded cytochrome $\mathrm{C}$ oxidase subunit 4 (COXIV) seems to contribute to a more unregulated respiration due to loss of ATP inhibition. Majority of energy for tumor transformations are mitochondrial origin. Differences in mitochondrial efficiency may be reflected in the progression of colorectal adenomatous polyps to adenocarcinomas. Here, we evaluate expression levels of COXIV isoform 1 (COXIV-1) and Mitochondrial (MT)-ATP synthase Subunit 6 (ATPase6) in adenomas of tubular, tubulovillous and villous tissues as compared to adenocarcinoma tissues.
\end{abstract}

Method: Both RT-qPCR and western blot techniques were used to assess COXIV-1 and ATPase6 expression levels in 42 pairs of patients' tissue samples. Protein carbonyl assay was performed to determine levels of oxidized proteins, as a measurement of ROS productions, in the tissue samples.

Results: Differential RNA expression levels of COXIV-1 and ATPase6 from whole tissues were observed. Interestingly, RNA expression levels obtained from mitochondrial for COXIV-1 were significantly decreased in tubulovillous, villous adenomas and adenocarcinoma, but not in the tubular-polyps. Moreover, mitochondrial ATPase6 RNA expression levels decreased progressively from adenopolyps to adenocarcinoma. In mitochondrial protein, expression levels of both genes progressively decreased with a three folds from adenomatous polyps to adenocarcinoma. Whilst the ATPase6 protein expression significantly decreased in adenocarcinoma compared to villous, conversely, the levels of oxidized carbonyl proteins were considerably increased from adenomatous polyps to adenocarcinoma.

Conclusion: Our findings provide evidence that decreased mitochondrial protein expression of COXIV-1 and ATPase 6 correlates with increased ROS production during colorectal adenomatous polyps' progression, suggesting the pivotal role of COXIV-1 in energy metabolism of colorectal cells as they progress from polyps to carcinoma.

Keywords: ATP synthase FO Subunit 6; COX subunit 4 isoform 1; Mitochondrial; Colorectal adenopolyps; Reactive oxygen species

Abbreviations CRC: Colorectal Cancer; Cp: Crossing Point; RTqPCR: Reverse Transcription Quantitative Polymerase Chain Reaction; COXIV-1: Cytochrome C Oxidase Subunit IV Isoform 1; MT-ATP6: ATP Synthase F0 Subunit 6; MT-DNA: Mitochondria DNA; TA: Tubular Adenoma; TV: Tubulovillous; V: Villous; CA: Cancer; ROS: Reactive Oxygen Species; DNPH: 2,4-Dinitrophenylhydrazine

\section{Introduction}

Despite great improvement in the treatment of colorectal cancer (CRC), this disease is still one of the most common malignancies worldwide and the third leading cause of cancer-related death in the United States [1,2]. The diagnosis and prognosis of patients with this disease remains disproportionally poor because the current clinicopathological parameters are not sufficient to precisely predict clinical outcome [3]. Therefore, there is a pressing need to explore appropriate molecular biomarkers to discriminate patients with different molecular pathological profiles, thus helping in elucidating pathways of progression from adenomas to carcinomas and perhaps, aid in individualize prognosis and therapeutic strategies.

In addition to nuclear genes, mitochondrial genome variations have also been evaluated in carcinogenesis due its important role in cancer cells type energy metabolism $[4,5]$. Most of the proteins that reside in the mitochondrion are nuclear gene products and these proteins are translated in the cytoplasmic compartment of eukaryotic cells and post-translationally, transported into the mitochondrion [6]. These proteins are involved in mitochondrial transcription, translation, mitochondrial lipid and heme synthesis; substrate oxidation by the Krebs cycle, electron transport and oxidative phosphorylation of the mitochondria. Moreover, approximately $90 \%$ of the proteins account for the mitochondrion mass and play roles in all aspects of mitochondrial function, and they are distributed in all four mitochondrial compartments (inner and outer mitochondrial 
Citation: Wallace L, Cherian AM, Adamson P, Bari S, Banerjee S, et al. (2018) Comparison of Pre- and Post-translational Expressions of COXIV-1 and MT-ATPase 6 Genes in Colorectal Adenoma-Carcinoma Tissues. J Carcinog Mutagen 9: 319. doi: $10.4172 / 2157-2158.1000319$

Page 2 of 9

membranes, matrix, and inter-membrane space) [5,7]. COXIV protein, one of the nuclear gene products plays a critical role in the assembly of COX and ATPase [7]. In addition, the COX complex has been suggested as a major regulator and control site for oxidative phosphorylation (OXPHOS) and reactive oxygen species (ROS) formation [8]. Regulation of OXPHOS and control of ROS production occurs due to proton slip at COX, allowing electrons to reduce oxygen without concomitant pumping of protons to the inter-membrane space thereby lowering mitochondrial efficiency $[9,10]$. Given that $C O X I V-1$ is responsible for allosteric regulation of COX activity via binding sites for both $\mathrm{ADP}$ and ATP, where the ATP/ADP ratio regulates its activity [11], a different expression of COXIV-1 may have impact on the mitochondrial ROS production due to a possible alteration of the membrane potential and may contribute to a more unregulated respiration due to loss of ATP inhibition [12]. It has been observed that the mitochondrial ATPase deficiency is associated with a decreased ability of cells in patients with cardiomyopathy to assemble ATPase complex proteins correctly $[13,14]$. In this study, we aimed to explore differential expression levels of COXIV and ATPase6 in colorectal adenomas tissues based on results from previous analyses of mitochondrial dysfunction involvement in tumor cells progression [15-17]. We hypothesized that the different RNA and protein expression levels of nuclear encoded $C O X I V-1$ and mitochondrial ATPase6 genes are important determinants of cellular ROS production and the subunits expression compositions may be correlated with colorectal adenomatous polyps progressive to adenocarcinomas. Realtime PCR and Western blot techniques were utilized to investigate the differential expression levels of COXIV and ATPase6 in colorectal adenomas tissues.

\section{Materials and Methods}

\section{Study samples}

Forty-two pairs of primary CRC tissues, from patients who underwent surgical resections, were obtained from the Southern Regional Cooperative Human Tissue Network. All studies were implemented under protocols approved by the Institutional Review Boards of Morehouse School of Medicine and the University of Alabama at Birmingham. Of the 42 paired tissue samples, 11 were from African-Americans, 29 were from Caucasians, and two were unknown demographics (Table 1). Fourteen of the samples were from men and 28 from women, with a mean age of 66 years [18].

\begin{tabular}{|c|c|c|}
\hline \multicolumn{2}{|l|}{ Demographics } & \multirow{2}{*}{$\begin{array}{l}\mathbf{n}^{*}(\%) \\
11 / 42(26 \%)\end{array}$} \\
\hline Race/ethinicity & African American & \\
\hline & Caucasian & $29 / 42(69 \%)$ \\
\hline & Other & $2 / 42(5 \%)$ \\
\hline \multirow[t]{2}{*}{ Gender } & Male & $14 / 42(33 \%)$ \\
\hline & Female & $28 / 42(67 \%)$ \\
\hline \multirow[t]{2}{*}{ Age } & Below 50 & $26 / 42(62 \%)$ \\
\hline & Above 50 & $16 / 42(38 \%)$ \\
\hline \multirow[t]{6}{*}{ Tumor type } & \multicolumn{2}{|l|}{ Adenomatous polyps } \\
\hline & Tubular Adenoma & $10 / 42(24 \%)$ \\
\hline & Tubulovillous Adenoma & $10 / 42(24 \%)$ \\
\hline & Villous Adenoma & $10 / 42(24 \%)$ \\
\hline & Cancer & \\
\hline & Adenocarcinoma & $12 / 42(28 \%)$ \\
\hline \multirow[t]{4}{*}{ Stages (Adenocarcinomas) } & Stage I & $4 / 12(33 \%)$ \\
\hline & Stage II & $2 / 12(2 \%)$ \\
\hline & Stage III & $2 / 12(2 \%)$ \\
\hline & Stage IV & $4 / 12(33 \%)$ \\
\hline \multirow[t]{5}{*}{ Tumor Region } & Left Colon & $2 / 42(5 \%)$ \\
\hline & Right Colon & $20 / 42(48 \%)$ \\
\hline & Transverse & $1 / 42(2 \%)$ \\
\hline & Sigmoid & $11 / 42(26 \%)$ \\
\hline & Rectum & $5 / 42(12 \%)$ \\
\hline
\end{tabular}


Citation: Wallace L, Cherian AM, Adamson P, Bari S, Banerjee S, et al. (2018) Comparison of Pre- and Post-translational Expressions of COXIV-1 and MT-ATPase 6 Genes in Colorectal Adenoma-Carcinoma Tissues. J Carcinog Mutagen 9: 319. doi: $10.4172 / 2157-2158.1000319$

Page 3 of 9

\begin{tabular}{|l|l|l|}
\hline & Metastatic & $1 / 42(2 \%)$ \\
\cline { 2 - 3 } & Unknown & $2 / 42(5 \%)$ \\
\hline$* 42$ paired tissue samples size (including tumor and normal surrounding tissues) \\
$* *$ Invasive carcinomas of stages I-IV were graded as adenocarcinoma (CA)
\end{tabular}

Table 1: Demographics of patients' tissue samples.

Tissue samples from patients with familial adenomatous polyposis (FAP), hereditary nonpolyposis syndrome, or inflammatory bowel disease were excluded. The selected samples including adjacent normal (N) tissues were from adenomatous polyps which comprise of tubular (T), tubulovillous (TV), villous (V) adenomas, and invasive carcinomas, which were graded as adenocarcinoma (CA) stages I-IV based on the degree of their glandular differentiation. Grade 1 adenocarcinoma is defined as well-differentiated, more than $95 \%$ of tumor composed of glands, including tubular adenocarcinomas; grade 2 is defined as moderately differentiated adenocarcinomas (50\%-95\% of tumor composed of glands), and grade 3 is defined as poorly differentiated carcinomas ( $49 \%$ or less of tumor composed of glands) as previously described [19].

All specimens were micro-dissected and adjacent normal tissues were defined as tissues excised from an area at least $5 \mathrm{~cm}$ away from the tumors. Tumor samples used in this study were diagnosed and histopathologically staged by pathologists according to the seventhedition Tumor-Node-Metastasis (TNM) staging system of the American Joint Committee on Cancer (AJCC). The selected samples were stabilized by snap-freezing immediately in cryovials and immersed in liquid nitrogen after excision and dissection and were transferred to $-80^{\circ} \mathrm{C}$ for long-term storage, as recommended for measurement of RNA and proteins as previously described [20].

\section{RNA extraction and quantitative-PCR analysis}

Total RNA extraction of tissue samples was performed with RNeasy Mini kits (Qiagen, Germantown, MD, USA). Briefly, frozen samples were cut on ice and washed with PBS twice before following the manufacturer's protocol. Following tissue homogenization, samples were placed in QIAshredder (Qiagen, Germantown, MD, US) columns to reduce viscosity. Purity of the RNA was assessed by $1 \%$ agarose gel electrophoresis and quantified with a Nanodrop ND-2000 spectrophotometer (Nanodrop Technologies, Wilmington, DE, US). For complementary DNA (cDNA) polymerization, RNA concentrations were adjusted to equal concentrations prior to analysis. Reverse transcription (RT) PCR was performed with iscript cDNA synthesis kits (BioRad, Hercules, CA, US) following the manufacturer's instructions, with $500 \mathrm{ng}$ of total RNA as a template and random hexamers as primers. Quantitative PCR was performed in a 480 Light cycler detector (Roche Diagnostics, Indianapolis, IN, US) with a SYBR green I master mix, $10 \mathrm{ng}$ of cDNA, and specific primers designed for the transcripts of nuclear-encoded COXIV-1, and MT encodedATPase6 genes (Table 2). Agarose gel electrophoresis was used to confirm primer specificity by checking for a single product of the expected length for each RT-qPCR transcript. The standard curves were generated for each primer set using serial dilutions of pooled cDNA to standardize variations in PCR reactions. In addition, after each run, melting curves were used to verify the melting temperature of the amplicons. All qPCR reactions were performed in triplicates. Second-derivative maximum analysis was used to calculate the crossing point $(\mathrm{Cp})$ values and the mRNA concentrations. For each gene, the value used for further calculations were the mean of the three replicates for each sample. To normalize against nonspecific variations in real-time PCR, the expression levels of three endogenous reference genes, glyceraldehyde-3-phosphate dehydrogenase (GAPDH), ubiquitin C (UBC), and $\beta$-actin (ATCB) (Table 2), in adjacent normal and tumor tissue samples were analyzed. The levels of RNA expression ratios in tumor tissue were based on comparisons of tumors and their adjacent normal tissues as previously described [20].

\begin{tabular}{|l|l|}
\hline \multirow{2}{|l|}{ Gene } & \multicolumn{1}{l}{ Primer Sequence } \\
\hline \multirow{2}{*}{ Nuclear/Mito-encoded genes } & \\
\hline \multirow{2}{*}{ MRC-complex V MT-ATP6 } & Forward: 5'-GAGTTGTATCGCATTAAGTTCAAGGAGAG-3' \\
\cline { 2 - 2 } & Reverse: 5'-ACACATAGTGCTTCTGCCACATGATAA-3' \\
\hline \multirow{2}{*}{ Housekeeping Genes } & Forward: 5'-TAGCCATACACAACACTAAAGGACGA-3' \\
\cline { 2 - 2 } & Reverse: 5'-GGGCATTTTTAATCTTAGAGCGAAA-3' \\
\hline \multirow{2}{*}{ ACTB } & Forward: 5'-TGCGTTACACCCTTTCTTGACA \\
\cline { 2 - 3 } & Reverse: 5'-GCAGGACTTCCTGTAACAATG-3' \\
\hline \multirow{2}{*}{ GAPDH } & Forward: 5'-GAAGGTGAAGGTCGGAGTC-3' \\
\cline { 2 - 2 } & Reverse: 5'-GAAGATGGTGATGGGATTTC-3' \\
\hline
\end{tabular}


Citation: Wallace L, Cherian AM, Adamson P, Bari S, Banerjee S, et al. (2018) Comparison of Pre- and Post-translational Expressions of COXIV-1 and MT-ATPase 6 Genes in Colorectal Adenoma-Carcinoma Tissues. J Carcinog Mutagen 9: 319. doi: $10.4172 / 2157-2158.1000319$

Page 4 of 9

$U B C$

Forward: 5'-ATTTGGGTCGCGGTTCTTG-3'

Reverse: 5'-TGCCTTGACATTCTCGATGG-3'

Table 2: Sequence of primers used for quantitative real time PCR.

\section{Western blot analysis}

The expressions of nuclear encoded, COXIV-1 and mitochondriaencoded ATPase6 polypeptides were analysed by SDS-PAGE and Western blot immunoassays in nuclear and mitochondrial proteins obtained from the studied colorectal adenomatous polyps and adenocarcinoma. Both normal surrounding tissues and Jurkat lysates (ATCC, Manassas, VA, USA) were used to show the differences in expression as colorectal adenomas progressed from normal surrounding tissue to adenocarcinoma (CA) tissue. The comparison of Jurkat lysates was used only in western blot studies to serve as a control for the COXIV-1 antibody. Briefly, total protein from nuclear and mitochondrial was isolated with the Mammalian Protein Extraction Reagent (Thermo Fisher Scientific, Waltham, MA USA) and mitochondrial protein isolation kit respectively as described previously [18]. Protein samples were resolved on 10\% stain-free SDSpolyacrylamide gels (Bio-Rad, Hercules, CA, USA). Proteins were transferred to Nitrocellulose membranes, blotted with a COXIV-1 and ATPase6 antibody and detected with a Super-Signal West Pico chemiluminescent substrate (Thermo Fisher Scientific, Waltham, MA USA). Superoxide Dismutase 2, Mitochondrial (SOD2) (Thermo Fisher Scientific, Waltham, MA USA) was used as a loading control, and blots were quantified by the relative optical density on the level of band intensity using the NIH Image J image analysis program. Additionally, the extracted mitochondrial protein was probed with actin and tubulin antibodies for confirmation of its purity.

\section{Protein carbonyl assay}

To determine if the levels of oxidized proteins correlated with the levels of both COXIV-1 and ATPase6 expression patterns in this study, spectrophotometric techniques were used to measure reactive protein carbonyl groups among samples. Generally, reactive oxygen species (ROS) react with histidine, arginine, lysine, and proline groups in proteins to produce carbonyl groups that react with 2,4dinitrophenylhydrazine (DNPH), leading to formation of stable dinitrophenyl hydrazone adducts [20-22]. DNPH was used to determine the amounts of reactive protein carbonyl contents in colorectal tissues using a protein carbonyl content assay kits (SigmaAldrich, St. Louis, MO USA). Briefly, DNA-free homogenates of samples $(100 \mu \mathrm{l})$ were placed in micro-centrifuge tubes, $100 \mu \mathrm{l}$ of 10 $\mathrm{mM} \mathrm{DNPH}$ in $2 \mathrm{M} \mathrm{HCl}$ was added, and the preparations were incubated at room temperature for $10 \mathrm{~min}$. The hydrazone derivatives were precipitated with $30 \mu \mathrm{l}$ of $100 \%$ of trichloroacetic acid. The preparations were vortexed, incubated on ice for $5 \mathrm{~min}$, and centrifuged at 13,000 g for $2 \mathrm{~min}$. The supernatant was removed, and $500 \mu \mathrm{l}$ of ice-cold acetone were added to the pellets, which were incubated at $-20^{\circ} \mathrm{C}$ for $5 \mathrm{~min}$ and centrifuged for $2 \mathrm{~min}$ at $13,000 \mathrm{~g}$. The pellets were dissolved in $6 \mathrm{M}$ guanidine hydrochloride and read at optical densities of $370 \mathrm{~nm}$. The absorptions of DNPH (nmol) incorporated as per milligram of proteins were calculated using the Lambert equation and an extinction coefficient 22,000.

\section{Statistical and data analysis}

Each experiment was repeated at least three times. Data were shown as Mean \pm S.E.M. and analyzed using Graph Pad Prism 7. Statistical comparisons between groups were analyzed using Student's $t$ test and Kruskal-Wallis one-way analysis test. A value of $\mathrm{p}<0.05$ was considered to indicate statistical significance. Calculations were made with Microsoft Excel/Access 2010. Charts and graphs were drawn with Graph Pad Prism 7.

\section{Results}

\section{Evaluation of COXIV-1 and ATP6 RNA expression levels obtained from whole tissue patient samples of colorectal adenomatous polyps and adenocarcinomas}

Evaluation of RNA levels against GAPDH using RT-qPCR based on a $<5 \%$ difference in median Cp-values between the normal and tumor tissues were performed within the studied groups among samples.

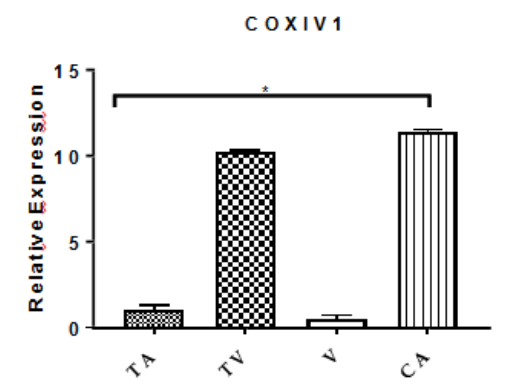

Figure 1: Comparison of the whole tissue RNA expression levels of COXIV-1 obtained from colorectal adenopolyps (TA: Tubular adenoma; TV: Tubulovillous adenoma; V: Villous adenoma) and adenocarcinoma (CA) patients' biopsies. Gene expression profile of COXIV-1 RNA was analyzed against Glyceraldehyde-3-phosphate dehydrogenase using RT-qPCR. Relative gene expression is expressed as the ratio of tumor tissues as compared to their paired normal surrounding tissue. Significant differences between groups were defined as ${ }^{*}$ with a $\mathrm{p}$-value $<0.05$ ( $\mathrm{N}=42$ samples).

There were highly significant nine folds increase in RNA expression levels from total whole tissue of nuclear encoded, $C O X I V-1$ gene observed in the adenomatous polyps of TV and CA when compared to relative low expression in $\mathrm{T}$ and $\mathrm{V}$ tissue samples (Figure 1). The ATPase6 RNA expression levels were highly significant in the adenomatous polyps of $\mathrm{V}$ with two and half folds higher than TV and almost two folds higher than $\mathrm{T}$ tissue samples. While the ATPase6 RNA expression levels in CA were two folds higher than the adenomatous polyps of TV and moderately higher than T (Figure 2). 
Citation: Wallace L, Cherian AM, Adamson P, Bari S, Banerjee S, et al. (2018) Comparison of Pre- and Post-translational Expressions of COXIV-1 and MT-ATPase 6 Genes in Colorectal Adenoma-Carcinoma Tissues. J Carcinog Mutagen 9: 319. doi: $10.4172 / 2157-2158.1000319$

Page 5 of 9

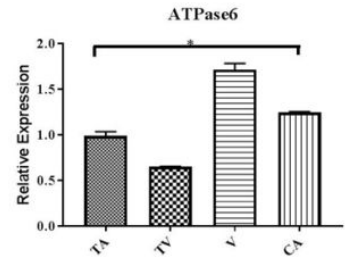

Figure 2: Comparison of the whole tissue RNA expression levels of $M T$-ATPase6 obtained from colorectal adenopolyps (TA: Tubular adenoma; TV: Tubulovillous adenoma; V: Villous adenoma) and adenocarcinoma (CA) patients' biopsies. Gene expression profile of ATPase6 RNA was analyzed using RT-qPCR. Relative gene expression is expressed as the ratio of tumor tissues as compared to their paired normal surrounding tissue. Significant differences between groups was defined as $^{*}$ with a p-value $<0.05 \quad(\mathrm{~N}=42$ samples).

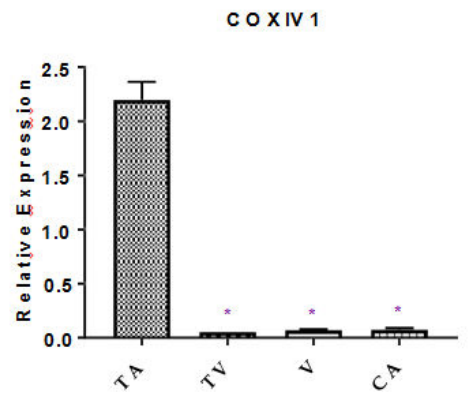

Figure 3: Comparison of the mitochondrial isolated RNA expression levels of COXIV-1 obtained from colorectal adenopolyps (TA: Tubular adenoma; TV: Tubulovillous adenoma; V: Villous adenoma) and adenocarcinoma (CA) patients' biopsies. Gene expression profile of COXIV-1 RNA was analyzed using RT-qPCR. Relative gene expression is expressed as the ratio of tumor tissues as compared to their paired normal surrounding tissue. Significant differences between groups was defined $\mathrm{as}^{\star}$ with a p-value $<0.05$ ( $\mathrm{N}=42$ samples).

These findings suggest functional differences in pre-translational transformation of the COXIV-1 and ATPase6 RNA expression levels in association to the progressive stages of colorectal adenomatous polyps. This result also indicates that high ATPase6 RNA levels may be highly involved in the transformational stage of villous adenoma. Moreover, this could also mean that the ATPase6 in total whole tissue RNA levels within colorectal adenomatous polyps of villous do not necessarily reflect the RNA levels in the stages of colorectal adenocarcinoma.

\section{Evaluation of COXIV-1 and ATPase 6 RNA and protein expression levels obtained from mitochondrial isolates of colorectal adenomatous polyps and adenocarcinoma tissues}

To gain insight into the critical role of both nuclear-encoded COXIV-1 and mitochondrial encoded ATPase6 RNA and protein expressions play within mitochondrial genome in association with the colorectal tumor progression, we evaluated mitochondrial RNA and protein expression levels obtained from the studied samples. Additionally, we also compared the results of the differential COXIV-1 and ATPase6 RNA expression levels obtained from whole tissue RNA lysates to determine the consistency with mitochondrial RNA and protein obtained from same tissue samples. We investigated these possibilities using RT-qPCR and Western blotting techniques.

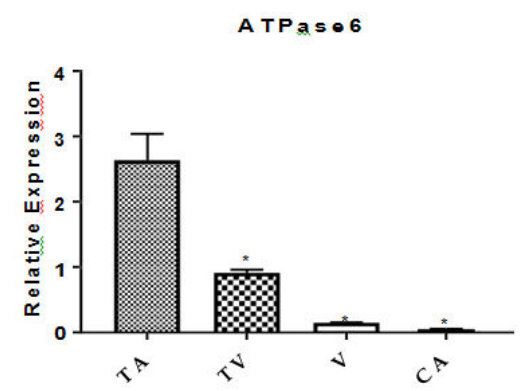

Figure 4: Comparison of the mitochondrial isolated RNA expression levels of ATPase6 obtained from colorectal adenopolyps (TA: Tubular adenoma; TV: Tubulovillous adenoma; V: Villous adenoma) and adenocarcinoma (CA) patients' biopsies. Gene expression profile of COXIV-1 RNA was analyzed against Glyceraldehyde-3-phosphate dehydrogenase using RT-qPCR. Relative gene expression is expressed as the ratio of tumor tissues as compared to their paired normal surrounding tissue. Significant differences between groups was defined as ${ }^{*}$ with a $\mathrm{p}$-value $<0.05$ ( $\mathrm{N}=42$ samples).

Interestingly, mitochondrial RNA expression levels of COXIV-1 decreased significantly in colorectal adenomatous polyps of TV, V and CA not in T tissue samples (Figure 3), while a progressive decreased of mitochondrial ATPase6 RNA expression levels were observed (Figure 4) among same tissue samples. Strikingly, in the Western blot assays, the protein expression levels in mitochondrial COXIV-1 rapidly decreased from adenomatous polyps ( $\mathrm{T}, \mathrm{TV}, \mathrm{V})$ to CA among tissue samples and Jurkat cells as a control (Figure 5). While the protein expression levels in mitochondrial ATPase6 appreciatively decreased three folds in CA tissue samples when compared to $\mathrm{V}$ of adenomatous polyps (Figure 5). Overall, the difference in the ATPase6 protein expression levels was found to be moderate among the adenomatous polyps of T, TV and V (Figure 5). This observation is highly important given that an assembly of mitochondrial ATPase6 gene are highly regulated by tissue-specific nuclear-encoded COXIV-1 expression.

The levels of the ATPase6 protein expressions moderately changes observed among normal and colorectal adenopolyps when compared to adenocarcinoma may indicate that the expressions of ATPase6 gene was not already surpassed by COXIV-1 in normal cell levels, where further substantiate decrease may not necessarily reflect on proportional effects on precancerous adenomatous polyps of T, TV, and $\mathrm{V}$ tissue samples. 
Citation: Wallace L, Cherian AM, Adamson P, Bari S, Banerjee S, et al. (2018) Comparison of Pre- and Post-translational Expressions of COXIV-1 and MT-ATPase 6 Genes in Colorectal Adenoma-Carcinoma Tissues. J Carcinog Mutagen 9: 319. doi: $10.4172 / 2157-2158.1000319$

Page 6 of 9

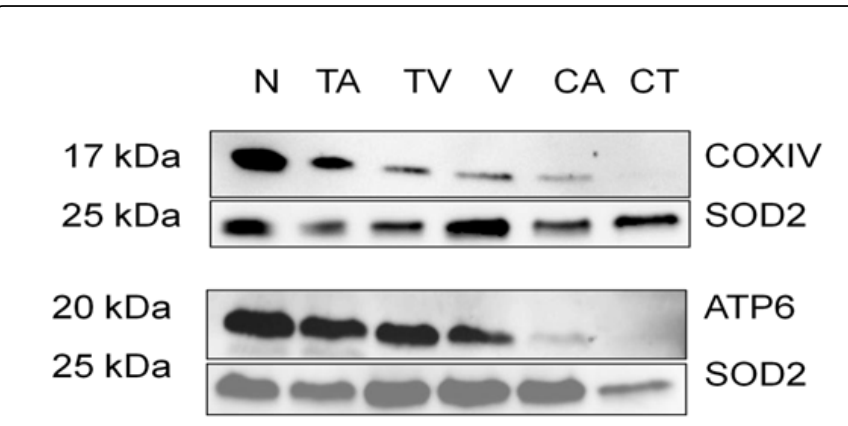

Figure 5: Mitochondria ATPase6 and COXIV-1 expression in colorectal tumors and normal tissue. (Upper figure) Lysates were analyzed by Western blot to examine the levels of COXIV-1 protein in normal tissues $(\mathrm{N})$, tubular adenoma tissues $(\mathrm{TA})$, tubulovillous adenoma tissues (TV), villous adenoma tissues (V), and Carcinoma tissues (CA). A control Jurkat cell lysate was also used for protein expression analysis. (Lower figure) Protein expression of ATPase6 analyzed through Western blot using the same parameters above. Each protein was normalized against Superoxide Dismutase 2 (SOD2).

\section{Potential effect of decrease of $C O X I V-1$ and $A T P a s e 6$ genes in post-translational in mitochondrial protein expressions in association to ROS productions in colorectal adenomatous polyps and adenocarcinoma tissues}

Next, we investigated the correlation of decreased COXIV-1 and ATPase6 expression in both whole tissues and mitochondrial isolates proteins obtained from colorectal adenomatous polyps and adenocarcinoma leading to less efficient mitochondrial coupling in association to increased ROS production among our studied tissue samples [20,22].

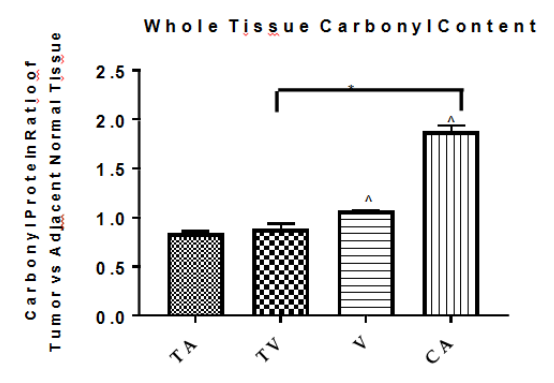

Figure 6: Comparison Levels of carbonyl reactive proteins in colorectal tissue as measured by spectrophotometry method. Ratios of carbonyl protein in whole tissue samples normalized against adjacent normal tissue samples; TA: Tubular adenoma; TV: Tubulovillous adenoma, V: Villous adenoma and CA: Adenocarcinoma. Significant differences between groups compared to cancer were calculated using $\operatorname{ANOVA}^{*}(\mathrm{P}<0.05)(\mathrm{n}=42$ pairs samples). Data are presented as Mean \pm S.E.M.

Results from the assay showed that carbonyl contents, which is reactive carbonyl proteins that formed through reaction with ROS as a characteristic of oxidative stress were progressively, but moderately increased from the among adenomatous polyps of T, TV, V and increased two folds in CA when compared to TA (Figure 6) in the protein obtained from whole tissue samples.

Similar results were observed in mitochondrial isolates proteins except carbonyl contents were lower in tubulovillous adenoma samples (Figure 7). This observation is similar to our previous work which carbonyl contents were measured from whole tissue protein lysates from the progressive stages of colorectal adenomatous polyps and adenocarcinoma samples [22]. Notably, analyzing the differences in expression levels, this is apparent to note that carbonyl proteins were increased in tissues that were derivatized. Also, the most noteworthy, perhaps, is this reactive carbonyl contents proteins assay results that measured the ROS production which were conversely proportional to mitochondrial encoded ATPase6 protein expression levels in same tissue samples (Figures 6, 7). Accordingly, decreased in the mitochondrial ATPase6 gene protein expression levels correlated with increased in the ROS productions among colorectal adenocarcinomas.

\section{Discussion}

Physiological processes including energy production, anabolic and catabolic metabolism, generation of reactive oxygen species and apoptosis has long been associated with mitochondrial function [23]. Malignancies of various types, development and, progression has been enhanced by mitochondrial dysfunction $[4,24]$. Past studies suggested that COX subunits and ATPase play a pivotal role in metabolic regulation and control of OXPHOS and ROS formation $[9,10]$. The COXIV-I isoform A is ubiquitously expressed and is responsible for allosteric regulation of COX activity via binding site for both ADP and ATP, where the ATP/ADP ratio regulates its activity [11], potentially having an impact on the mitochondrial ROS production due to membrane potential alterations [11,24-26] and its precise function is yet to be fully determined. Moreover, previous studies $[27,28]$ indicated that the ATPase deficiency is associated with a decreased ability of cells to assemble correctly the ATPase complex and that the COXIV isoform setup loses the control over metabolic rate as ATP/ADP ratio decreases and metabolic rate increases [29].

This study examined the gene expression of COXIV-1 and ATPase6 in relative to precancerous, adenomatous polyps and adenocarcinoma, of the colorectal tissues. In total RNA isolated from whole tissue samples and the observed RNA expression levels of the COXIV-1 were significantly higher in tubulovillous adenoma and adenocarcinoma, while the ATPase6 RNA expression levels were lower in tubular and tubulovillous adenoma and highly expressed in villous adenoma and moderately expressed in adenocarcinoma. In mitochondrial RNA, expression levels of COXIV-1 were significantly decreased in tubulovillous, villous adenoma and adenocarcinoma, but not in the tubular tissue. While progressive decreases were observed in ATPase6 RNA expression levels from tubular, tubulovillous and villous adenomas to adencarcinoma.

Accordingly, in the Western blot assays, the protein expression levels in COXIV-1 rapidly decreased progressively from tubular, tubulovillous and villous adenoma to adenocarcinoma among the tissue samples and in Jurkat cancer cells that was used as a control. While ATPase6 appreciatively decreased three folds in adenocarcinoma when compared to villous adenoma. Additionally, various expression levels of ATPase6 were moderately observed among the tubular, tubulovillous and villous adenoma. Moreover, results from 
Citation: Wallace L, Cherian AM, Adamson P, Bari S, Banerjee S, et al. (2018) Comparison of Pre- and Post-translational Expressions of COXIV-1 and MT-ATPase 6 Genes in Colorectal Adenoma-Carcinoma Tissues. J Carcinog Mutagen 9: 319. doi:

the protein carbonyl assay that measured carbonyl contents, which is the reactive carbonyl proteins that formed through reaction with ROS as a characteristic of oxidative stress, were moderately increased in the tubular to villous adenoma and two folds increased in adenocarcinoma when compared with tubular adenoma (Figures 6 and 7). This result is consistent with previous findings while working with the whole proteins lysates [22]. Perhaps, most importantly is the observed elevation of the expression of carbonyl contents level in adenocarcinoma as an indicator of reactive oxygen species to correlate with decrease in ATPase6 protein expression levels of same tissues sample of adenocarcinoma. These findings suggest that the observed decrease in $C O X I V-1$ may have negatively impacted ATPase 6 protein expression levels in mitochondrial genome which may have resulted in the elevation of ROS production levels in the colorectal adenocarcinoma.

Mitochondrial Carbonyl Content

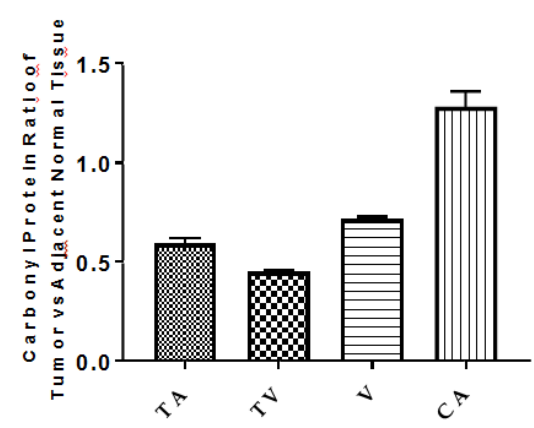

Figure 7: Comparison levels of carbonyl reactive proteins in colorectal tissue as measured by spectrophotometry method. Ratios of carbonyl protein in whole tissue samples normalized against adjacent normal tissue samples; TA: Tubular adenoma; TV: Tubulovillous adenoma, V: Villous adenoma, and CA: Adenocarcinoma. Significant differences between groups compared to cancer were calculated using ANOVA $^{*}(\mathrm{P}<0.05)(\mathrm{n}=42$ pairs samples). Data are presented as Mean \pm S.E.M.

The ATPase6 protein encoded by the mitochondrial genome is a component of a large enzyme called ATP synthase which catalyzes the final step of oxidative phosphorylation [30,31]. The physiological role of ATPase6 effects on cell ATP production is well known, given that the cellular content of ATPase differs in mammalian tissues and this may vary also during oncogenetic development and progression of different cancer cells type. Furthermore, relative to OXPHOS complexes, physiologically, ATPase is generally down-regulated about ten-folds [32]. However, its biological functions in association with nuclear encoded $C O X I V-1$ gene in terms of regulation and assembly of its biosynthesis in relative to the progression of colorectal adenomatous polyps is still not very well understood. The observed decreased in ATPase6 protein expression levels which correlated with increased mitochondrial ROS production from precancerous adenomas to adenocarcinoma in this study may be more relevant to its involvement in decreased COXIV-1 proteins as a ATPase6- specific assembly protein.
Indeed, a past study by Zhang et al. [7] indicated that COXIV expression was elevated in CRC tissues when compared with adjacent noncancerous tissues at both RNA and protein levels, but the specific COXIV isoforms RNA and protein actual expression levels in colorectal early adenomas have been poorly investigated. There are various COX subunit isoforms with different functionalities in association with specific tissue types. This study mainly focused on the expression levels of COXIV-1 in relationship with ATPase6 and its effect on ROS production in the progressive stages of precancerous tubular, tubulovillous, villous adenoma and adenocarcinoma. Our results are in agreements with other studies that have reported decrease expression of nuclear genes encoding of four OXPHOS subunits; COXIV, COXVa, SD30, and SD7 of mtDNA in depleted fibroblasts. Marusich et al. [33] and Chevallet et al. [34] reported differential levels of decreased respiratory complex subunits, translation apparatus (particularly mitochondria ribosomal proteins), and ion and protein import systems, such as membrane proteins, that were found in $143 \mathrm{~B} \rho^{\circ}$ cells when compared to wild-type cells. Thus the capacity of MT-complex IV to transfer electrons and the ability of MTcomplex V to receive electrochemical gradient energy could affect ATP synthesis. This effect could lead to the accumulation of electrons resulting from excessive leakage of oxygen as free radicals ultimately increases ROS production in cells.

Furthermore, a COXIV subunit has been suggested to play pleiotropic role by providing a mechanism of maintaining the efficiency of respiration under conditions of reduced $\mathrm{O}_{2}$ availability and may lead to the initial adaptive response to hypoxia $[4,35]$. A COXIV-1 has been shown to be a key regulatory subunit of mammalian cytochrome $c$ oxidase by adjusting energy production to match the cellular energy requirements of cancer cells $[4,36,37]$. This metabolic mechanism in cancer cells is suggested to fuel proliferation primarily by a shift to anaerobic glycolysis termed as Warburg effect $[4,38,39]$. Nevertheless, recent studies have indicated that the metabolic characteristics of glioma cells are heterogeneous and there are specific differences in glucose uptake and dependency in glioma cell lines [4,40-44]. Moreover, Marin-Valencia at al. suggested that genetic diversity in human glioblastomas may exhibit a high rate of glucose uptake of either glycolysis or mitochondrial glucose oxidation as an energy source [45]. Therefore, it could be inferred that the differentiation status of regulatory subunit of COXIV-1 protein in the mitochondrial genome in relative to the ATPase6 protein activities could be related to energy metabolism and redox homeostasis of the early CRC cells. Most importantly, it is possible that these observed changes in COXIV-1 protein expression in the mitochondrial genome in this study characterized an aspect of the pleiotropic response of the colorectal precancerous cells to progression signals or initiating these processes.

To the best of our knowledge, there are limited pre- and posttranslational studies of nuclear-encoded $C O X I V-1$ and mitochondrial encoded ATPase6 gene expressions linking to the elevation of ROS production in precancerous colorectal adenomatous polyps (tubular, tubulovillous and villous) and adenocarcinoma. Our findings demonstrate an involvement of decrease in mitochondrial RNA protein expressions of COXIV-1 and ATPase6 gene, and conversely, increase ROS production during the colorectal adenomatous polyps progression. Collectively, data in this study suggests that the observed progressive decrease in nuclear-coded COXIV-1 RNA and protein expressions in the mitochondrial genome play a pivotal role in the assembly and disassembly of post-translational expression of ATPase6 gene functions that may negatively alter ATP synthase complex, hence, 
Citation: Wallace L, Cherian AM, Adamson P, Bari S, Banerjee S, et al. (2018) Comparison of Pre- and Post-translational Expressions of COXIV-1 and MT-ATPase 6 Genes in Colorectal Adenoma-Carcinoma Tissues. J Carcinog Mutagen 9: 319. doi: $10.4172 / 2157-2158.1000319$

Page 8 of 9

increase in the ROS production during the progression of colorectal adenomatous polyps to adenocarcinoma. This study warrants further investigation onto the impact of alterations of ATPase6 gene functionality as a result of lower expression of COXIV-1 protein in the mitochondrial genome in the precancerous stages of other cancer cell types.

\section{Declarations}

The authors declare no conflict of interest.

\section{Author's Contributions}

Conception and design: L. Wallace, FO. Aikhionbare; Development of methodology: L. Wallace, FO. Aikhionbare; Acquisition of data: L. Wallace, S. Banerjee, AM Cherian; Analysis and interpretation of data: FO. Aikhionbare, L. Wallace; Writing: FO. Aikhionbare, L. Wallace, X. Yao, P. Adamson, M. Flood, M Simien, S. Bari

\section{Acknowledgements}

This work was supported by grant; NIH-NIGMS (GM122669 and GM099663) awarded to Dr. Felix O Aikhionbare and partly supported by MBRC Program from the National Institute of Minority Health and Health Disparities, Grant Number 8G12MD007602. To those investigators whose meritorious works could not be cited due to space limitations, we honestly apologize. This study content is solely the responsibility of the authors and does not necessarily represent the official views of the NIH-NIMHD and NIGMS.

\section{References}

1. Jemal A, Bray F, Center MM, Ferlay J, Ward E, et al. (2017) Global cancer statistics. CA Cancer J Clin 61: 69-90.

2. Stec R, Plawski A, Synowiec A, Maczewski M, Szczylik C (2010) Colorectal cancer in the course of familial adenomatous polyposis syndrome ("de novo" pathogenic mutation of APC gene): case report, review of the literature and genetic commentary. Arch Med Sci 6: 283-287.

3. Siegel RL, Miller KD, Jemal A (2017) Colorectal Cancer Mortality Rates in Adults Aged 20 to 54 Years in the United States, 1970-2014. JAMA 318: 572-574.

4. Oliva CR, Markert T, Gillespie GY, Griguer CE (2015) Nuclear-encoded cytochrome $\mathrm{c}$ oxidase subunit 4 regulates BMI1 expression and determines proliferative capacity of high-grade gliomas. Oncotarget 6 : 4330-4344.

5. Sanz-Pamplona R, Berenguer A, Cordero D, Riccadonna S, Solé X, et al. (2012) Clinical value of prognosis gene expression signatures in colorectal cancer: a systematic review. PLoS One 7: e48877.

6. Glick B, Schatz G (1991) Import of proteins into mitochondria. Annu Rev Genet 25: 21-44.

7. Zhang K, Chen Y, Huang X, Qu P, Pan Q, et al. (2016) Expression and clinical significance of cytochrome $\mathrm{c}$ oxidase subunit IV in colorectal cancer patients. Arch Med Sci 12: 68-77.

8. Kadenbach B, Huttemann M, Arnold S, Lee I, Bender E (2000) Mitochondrial energy metabolism is regulated via nuclear-coded subunits of cytochrome c oxidaseFree. Radic Biol Med 29: 211-221.

9. Frank V, Kadenbach B (1996) Regulation of the H_/e_stoichiometry of cytochrome $c$ oxidase from bovine heart by intramitochondrial ATP/ADP ratios. FEBS Lett 382: 121-124.

10. Kadenbach B (2003) Intrinsic and extrinsic uncoupling of oxidative phosphorylation. Biochim Biophys Acta 1604: 77-94.
11. Arnold S (2012) The power of life- cytochrome c oxidase takes center stage in metabolic control, cell signalling and survival. Mitochondrion 12: $46-56$.

12. Horvat S, Beyer C, Arnold S (2006) Effect of hypoxia on the transcription pattern of subunit isoforms and the kinetics of cytochrome $c$ oxidase in cortical astrocytes and cerebellar neurons. J Neurochem 99: 937-951.

13. Houstek J, Klement $\mathrm{P}$, Hermanska J, Houstkova H, Hansikova H, et al. (1995) Altered properties of mitochondrial ATP-synthase in patients with a $T \rightarrow G$ mutation in the ATPase 6 (subunit a) gene at position 8993 of mtDNA. Biochim. Biophys. Acta 1271: 349-357.

14. Nijtmans LG, Henderson NS, Attardi G, Holt IJ (2001) Impaired ATP synthase assembly associated with a mutation in the human ATP synthase subunit 6 gene. J Biol Chem 276: 6755-6762.

15. Yu M (2012) Somatic mitochondrial DNA mutations in human cancers. Adv Clin Chem 57: 99-138.

16. Chatterjee A, Dasgupta S, Sidransky D (2011) Mitochondrial subversion in cancer. Cancer Prev Res (Phila) 4: 638-654.

17. Houshmand M, Montazeri M, Kuchekian N, Noohi F, Nozar G (2011) Is 8860 variation a rare polymorphism or associated as a secondary effect in HCM disease? Arch Med Sci 7: 242-246.

18. Wallace L, Mehrabi S, Bacanamwo M, Yao X, Aikhionbare FO (2016) Expression of mitochondrial genes MT-ND1, MT-ND6, MT-CYB, MTCOI, MT-ATP6, and 12S/MT-RNR1 in colorectal adenopolyps. Tumour Biol 37: 12465-12475.

19. Fleming M, Ravula S, Tatishchev SF, Wang HL (2012) Colorectal carcinoma: Pathologic aspects. J Gastrointest Oncol 3: 153-173.

20. Beal MF (2002) Oxidatively modified proteins in aging and disease. Free Radical Biology and Medicine 32: 797-803.

21. Levine RL, Garland D, Oliver CN, Amici A, Climent I, et al. (1990) Methods in enzymology oxygen radicals in biological systems part B: oxygen radicals and antioxidants. Determination of carbonyl content in oxidatively modified proteins, New York Academic Press, United States pp. 464-478.

22. Mehrabi S, Wallace L, Cohen S, Yao X, Aikhionbare FO (2015) Differential Measurements of Oxidatively Modified Proteins in Colorectal Adenopolyps. Int J Clin Biostat Biom 6: 288- 299.

23. Koppenol WH, Bounds PL, Dang CV (2011) Otto Warburg's contributions to current concepts of cancer metabolism. Nat Rev Cancer 11: 325-337.

24. Li Y, Park JS, Deng JH, Bai Y (2006) Cytochrome c Oxidase Subunit IV is Essential for Assembly and Respiratory Function of the Enzyme Complex. J Bioenerg Biomembr 38: 283-291.

25. Fukuda R, Zhang H, Kim JW, Shimoda L, Dang CV, et al. (2007) HIF-1 Regulates Cytochrome Oxidase Subunits to Optimize Efficiency of Respiration in Hypoxic Cells. Cell 129: 111-122.

26. Kadenbach B, Hüttemann M (2015) The subunit composition and function of mammalian cytochrome c oxidase. Mitochondrion 24: 64-76.

27. Houstek J, Mracek T, Vojtiskova A, Zeman J (2004) Mitochondrial diseases and ATPase defects of nuclear origin. Biochim Biophys Acta 1658: 115-121.

28. Nijtmans LG, Henderson NS, Attardi G, Holt IJ (2001) Impaired ATP synthase assembly associated with a mutation in the human ATP synthase subunit 6 gene. J Biol. Chem 276: 6755-6762.

29. Schiffer TA, Peleli M, Sundqvist ML, Ekblom B, Lundberg JO, et al. (2016) Control of human energy expenditure by cytochrome $\mathrm{c}$ oxidase subunit IV-2. Am J Physiol Cell Physiol 311: C452-C461.

30. Anderson S, Bankier AT, Barrell BG, de Bruijn MH, Coulson AR, et al. (1981) Sequence and organization of the human mitochondrial genome. Nature 290: 457-465.

31. Andrews RM, Kubacka I, Chinnery PF, Lightowlers RN, Turnbull DM, et al. (1999) Reanalysis and revision of the Cambridge reference sequence for human mitochondrial DNA. Nat Genet 23: 147.

32. Houstek J, Kopecky J, Baudysova M, Janikova D, Pavelka S, et al. (1990) Differentiation of brown adipose tissue and biogenesis of thermogenic 
Citation: Wallace L, Cherian AM, Adamson P, Bari S, Banerjee S, et al. (2018) Comparison of Pre- and Post-translational Expressions of COXIV-1 and MT-ATPase 6 Genes in Colorectal Adenoma-Carcinoma Tissues. J Carcinog Mutagen 9: 319. doi: $10.4172 / 2157-2158.1000319$

Page 9 of 9

mitochondria in situ and in cell culture. Biochim. Biophys. Acta 1018: 243-247.

33. Marusich MF, Robinson BH, Taanman JW, Kim SJ, Schillace R (1997) Expression of mtDNA and nDNA encoded respiratory chain proteins in chemically and genetically-derived Rho0 human fibroblasts: a comparison of subunit proteins in normal fibroblasts treated with ethidium bromide and fibroblasts from a patient with mtDNA depletion syndrome. Biochim. Biophys. Acta 1362: 145-159.

34. Chevallet M, Lescuyer P, Diemer H, van Dorsselaer A, Leize-Wagner E, et al. (2006) Alterations of the mitochondrial proteome caused by the absence of mitochondrial DNA: a proteomic view. Electrophoresis 27: 1574-1583.

35. Fukuda R, Zhang H, Kim JW, Shimoda L, Dang CV, et al. (2007) HIF-1 regulates cytochrome oxidase subunits to optimize efficiency of respiration in hypoxic cells. Cell 129: 111-122.

36. Arnold S, Lee I, Kim M, Song E, Linder D, et al. (1997) The subunit structure of cytochrome-c oxidase from tuna heart and liver. Eur J Biochem 248: 99-103.

37. Napiwotzki J, Shinzawa-Itoh K, Yoshikawa S, Kadenbach B (1997) ATP and ADP bind to cytochrome $\mathrm{c}$ oxidase and regulate its activity. Biol Chem 378: 1013-1021.

38. Warburg O (1956) On respiratory impairment in cancer cells. Science 124: $269-270$.
39. Warburg O (1956) On the origin of cancer cells. Science 123: 309-314.

40. Oliva CR, Moellering DR, Gillespie GY, Griguer CE (2011) Acquisition of chemoresistance in gliomas is associated with increased mitochondrial coupling and decreased ROS production. PloS one 6: e24665.

41. Oliva CR, Nozell SE, Diers A, McClugage SG, Sarkaria JN, et al. (2010) Acquisition of temozolomide chemoresistance in gliomas leads to remodeling of mitochondrial electron transport chain. J Biol Chem 285: 39759-39767.

42. Elstrom RL, Bauer DE, Buzzai M, Karnauskas R, Harris MH, et al. (2004) Akt stimulates aerobic glycolysis in cancer cells. Cancer Res 64: 38923899.

43. Griguer CE, Oliva CR, Gillespie GY (2005) Glucose metabolism heterogeneity in human and mouse malignant glioma cell lines. J Neurooncol 74: 123-133.

44. Saga I, Shibao S, Okubo J, Osuka S, Kobayashi Y, et al. (2014) Integrated analysis identifies different metabolic signatures for tumor-initiating cells in a murine glioblastoma model. J Neurooncol 16: 1048-1056.

45. Marin-Valencia I, Yang C, Mashimo T, Cho S, Baek H, et al. (2012) Analysis of tumor metabolism reveals mitochondrial glucose oxidation in genetically diverse human glioblastomas in the mouse brain in vivo. Cell Metab 15: 827-837. 